\title{
Differential Roles of Dopamine DI and D2 Receptors in the Nucleus Accumbens in Attentional Performance on the Five-Choice Serial Reaction Time Task
}

\author{
Marie-Astrid Pezze', Jeffrey W Dalley' and Trevor W Robbins*,' \\ 'Department of Experimental Psychology, University of Cambridge, Cambridge, UK
}

\begin{abstract}
Nucleus accumbens (NAC) dopamine may play a role in attentional and executive processes, as it modulates cortico-limbic inputs, including afferents from the prefrontal cortex. The present study examined the role of NAC dopamine DI and D2 receptors in visual attentional processes and response control in rats as assessed in the five-choice serial reaction time task (5CSRT). Rats were trained to detect the location of brief $(0.5 \mathrm{~s})$ visual targets presented randomly in an array of five apertures to receive food reward. They were tested after bilateral infusions of a DI receptor antagonist (SCH 23390) and agonist (SKF 38393) and a D2 receptor antagonist (sulpiride) and agonist (quinpirole) into the NAC. While intra-NAC SCH 23390 decreased accurate responding and increased response

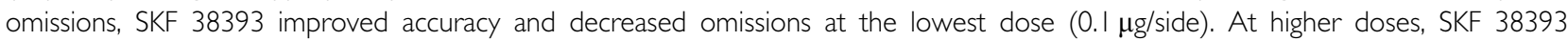
increased premature 'impulsive' responding. Sulpiride impaired the attentional accuracy of responding and slowed the latency to collect the earned food reward. By contrast, intra-NAC infusions of quinpirole did not significantly affect attentional accuracy, but increased perseverative responding. Optimal performance on the 5CSRT depends on both DI and D2 receptors in the NAC, but they modulate different aspects of performance. DI receptor agents had more selective effects on attentional accuracy while D2 receptor stimulation did not affect accuracy or premature responses, but enhanced perseverative responding. The data are discussed in terms of the different functions of NAC dopamine receptors in the processing of information from its different cortico-limbic inputs. Neuropsychopharmacology (2007) 32, 273-283. doi: 10.1038/sj.npp. I 30 I073; published online 12 April 2006
\end{abstract}

Keywords: attention; dopamine; impulsivity; nucleus accumbens; DI receptors; D2 receptors

\section{INTRODUCTION}

The nucleus accumbens (NAC) receives major inputs from cortico-limbic structures, including the medial prefrontal cortex (mPFC) (Groenewegen et al, 1999), but it is poorly understood how the NAC mediates behavioral functions known to depend on the PFC. These 'executive' processes include working memory (Goldman-Rakic et al, 1990; Floresco et al, 1996), attention to action, and inhibitory response control (Robbins, 2000), impairments in which may contribute to clinical disorders such as attention deficit/ hyperactivity disorder and schizophrenia (see Chudasama and Robbins, 2004a).

Excitotoxic lesions of different portions of the mPFC in rats produce dissociable deficits in performance in terms of

\footnotetext{
*Correspondence: Dr TW Robbins, Department of Experimental Psychology, University of Cambridge, Downing Street, Cambridge CB2 3EB, UK, Tel: + 441223 765291,

E-mails: map46@cam.ac.uk or twr2@cam.ac.uk

Received 19 October 2005; revised 9 February 2006; accepted 9 February 2006

Online publication: 6 March 2006 at http://www.acnp.org/citations/ Npp022706050635/default.pdf
}

accuracy and premature or perseverative responding on the five-choice serial reaction time task (5CSRT) (Muir et al, 1996; Passetti et al, 2002; Chudasama et al, 2003), a test which recruits attentional and inhibitory control processes (Robbins, 2002). Moreover, intra-mPFC infusions of the D1 partial agonist SKF 38393 selectively improve attentional accuracy on this task in poorly performing rats, whereas the D1 receptor antagonist SCH 23390 has the opposite effect in highly performing animals (Granon et al, 2000). A number of studies have indicated that performance on the 5CSRT is also influenced by excitotoxic lesions of the striatum (Christakou et al, 2004; Rogers et al, 2001; Cole and Robbins, 1987, 1989; Baunez and Robbins, 1999) suggesting the involvement of defined fronto-striatal circuitry in 5SCRT performance (Christakou et al, 2004). Moreover, there is evidence that dopamine (DA) depletion from the dorsal (Baunez and Robbins, 1999) or ventral striatum (Cole and Robbins, 1987, 1989) also modulates 5CSRT performance. However, there has been little investigation to date on the role of striatal D1 and D2 receptors.

Two studies, using a different paradigm for assessing attentional functions have reported detrimental effects following inactivation of GABA synthesis in the NAC 
(Miner and Sarter, 1999) or intra-NAC infusion of the mixed DA receptor antagonist cis-flupenthixol (Himmelheber et al, 2000). It has also been demonstrated that manipulation of NAC DA function by intra-NAC infusions of D1 or D2 receptor agents modulate the rate or vigor of responding with conditioned reinforcement or to rewardpredictive cues (Taylor and Robbins, 1986; Wolterink et al, 1993; Nicola et al, 2005), sometimes interpreted as 'incentive salience' (Berridge and Robinson, 1998; Wyvell and Berridge, 2000). Yet, it is unclear how such rateincreasing effects on behavior might correspond to possible changes in attentional function.

The integration of information in the NAC by DA-ergic mechanisms depends critically on the class of DA receptor. While D2 receptors in the NAC attenuate mPFC inputs (O'Donnell and Grace, 1994; Brady and O'Donnell, 2004), DA D1 receptors sustain the state-dependent excitability of medium spiny neurons (O'Donnell, 2003). Recently, Goto and Grace (2005) have shown that DA in the NAC selectively modulates synaptic input from the hippocampus and the mPFC through D1 and D2 receptors, respectively, corresponding to different aspects of goal-directed behavior.

Therefore, in the present study, we examined the role of D1 and D2 receptor-mediated processes in the NAC of rats in 5CSRT performance. For this purpose, we used intraNAC micro-infusions of the D1 receptor antagonist SCH 23390 and the partial D1 agonist SKF 38393, as well as of the D2 receptor antagonist sulpiride and the D2 agonist quinpirole into the NAC over a range of doses.

\section{MATERIALS AND METHODS}

\section{Subjects}

The subjects were 66 male hooded Lister rats (Charles River, UK), housed in pairs under temperature-controlled conditions and alternating 12-h-light-12-h-dark cycle (lights off at $0700 \mathrm{~h}$ ). They were maintained at $80-85 \%$ of their free feeding weight by restricting access to laboratory chow (Purina, UK) to $18 \mathrm{~g} /$ day per rat. Water was provided ad libitum. All procedures were conducted in accordance with the requirements of the UK Animals (Scientific Procedures) Act 1986 and in accordance with local institutional guidelines.

\section{CSRT}

Apparatus. A detailed description of the nine-hole apparatus has been provided previously (Carli et al, 1983; Robbins et al, 1993). Briefly, eight $25 \times 25 \times 25 \mathrm{~cm}^{3}$ nine-hole boxes (Paul Fray Ltd, UK) were used, each contained within a ventilated and sound-attenuated chamber and illuminated by a $3-\mathrm{W}$ house light. Nine evenly spaced square holes $\left(2.5 \times 2.5 \times 4 \mathrm{~cm}^{3}\right)$ containing a 3 -W light were set into the curved aluminum wall at the rear of the box, $2 \mathrm{~cm}$ above the grid floor. A metal cap blocked hole numbers $2,4,6$, and 8 . An infrared beam located at the entrance to each hole, enabled detection of nose-poke responses. Every other hole was blocked, so that only five of the holes were accessible. A food magazine, into which food pellets (Noyes dustless pellets, $45 \mathrm{mg}$; Sandown Scientific, UK) could be dispensed, was located in the middle of the opposite wall. An infrared beam located horizontally across the entrance to the magazine allowed recording of entries into the magazine. The distance from the center hole at the rear of the box and the magazine was $25 \mathrm{~cm}$. The apparatus and on-line data collection were controlled by means of an Archimedes computer system with software written in ARACHNID (Paul Fray, Cambridge, UK).

Training. Rats were trained on the 5CSRT to a stable level of performance as described previously (Granon et al, 2000). Briefly, the rats initiated a trial by nose poking into the magazine. After an intertrial interval (ITI), arbitrarily fixed at $5 \mathrm{~s}$, the light at the rear of one of the apertures was presented randomly in one of the five possible locations ( 1 , $3,5,7$, and 9) for a short period ( $0.5 \mathrm{~s})$. Responses in this aperture within a limited time of illumination of the hole (limited hold period) were recorded as correct responses and were rewarded by the delivery of a food pellet to the magazine. Responses in a nonilluminated hole (incorrect responses), failure to respond within the $5 \mathrm{~s}$ limited hold period (omission), and responses in one of the apertures during the ITI (premature or impulsive responses) were also recorded and punished by a $5 \mathrm{~s}$ time-out period, during which the illumination of the chamber was switched off. This behavior can be viewed as impulsive as it meets the definition of inappropriate responding performed without apparent foresight of the adverse outcome. Repeated responding in any hole during the presentation of the light stimulus or during the limited hold period was classified as perseverative responding and, while recorded, was not punished. The stimulus duration was $3 \mathrm{~s}$ in the initial training sessions and then progressively reduced to the final duration used during testing $(0.5 \mathrm{~s})$, depending on the rats' individual performance. Each session consisted of 100 trials, which lasted approximately $30 \mathrm{~min}$. After an average of 30 daily sessions, rats reached the criterion of stable preoperative performance when they made around $80 \%$ correct responses with fewer than $20 \%$ omissions. Task performance was reflected in the following behavioral measures:

- Choice accuracy: proportion of correct responses to total correct + incorrect responses, expressed as a percentage.

- Omissions: proportion of omission trials to total trials (ie correct + incorrect + omissions trials), expressed as a percentage.

- Premature responses: proportion of premature responses to total trials, expressed as a percentage.

- Perseverative responses: proportion of perseverative responses to total trials, expressed as a percentage.

- Correct response latency: mean time between stimulus onset and a nose poke in the correct hole.

- Magazine latency: mean time between a nose poke in the correct hole and food collection from the magazine.

\section{Surgery}

Rats were anesthetized with ketamine (Ketaset, $100 \mathrm{mg} / \mathrm{kg}$ i.p.; Vet Drug, Bury St, Edmunds, UK) and xylazine (Rompun $10 \mathrm{mg} / \mathrm{kg}$ i.p.; Vet Drug) and secured in a stereotaxic frame with the incisor bar set at $-3.3 \mathrm{~mm}$ relative to the interaural line. Bilateral guide cannulae 
(Plastics One, UK) consisting of a plastic body holding two 21 gauge metal tubes $3.8 \mathrm{~mm}$ apart and projecting $4 \mathrm{~mm}$ from the pedestal were implanted $5 \mathrm{~mm}$ above the injection site in the NAC core at the following coordinates: anteroposterior, $+1.5 \mathrm{~mm}$ from bregma; lateromedial, $\pm 1.9 \mathrm{~mm}$ from midline; and dorsoventral, $-2.2 \mathrm{~mm}$ from skull. For experiments testing the site specificity of infusion effects, similar cannulae, with metal tubes $5 \mathrm{~mm}$ apart and projecting $4 \mathrm{~mm}$ from the pedestal, were implanted $2 \mathrm{~mm}$ above the injection site in the dorsolateral striatum at the following coordinates: anteroposterior, $+1.5 \mathrm{~mm}$ from bregma; lateromedial, $\pm 2.5 \mathrm{~mm}$ from midline; and dorsoventral, $-3 \mathrm{~mm}$ from skull (Paxinos and Watson, 1998). Cannulae were secured to the skull with dental acrylic and stainless-steel screws, and a wire stylet occluded the guide to maintain patency. After surgery, the animals were housed individually and allowed 5-7 days recovery.

\section{Microinfusion Procedure}

Following re-establishment of stable postoperative performance over five to seven sessions, rats were habituated to the infusion procedure with one control vehicle infusion $(0.9 \% \mathrm{NaCl})$. Infusion studies were run in 3-day cycles, starting with a baseline session. The following day, the rats received a drug or vehicle infusion 5 min before testing in the 5CSRT. On the third day, animals were not tested and remained in their home cage. In all within-subjects studies, the different drug doses were tested in a counterbalanced order, if possible, in a Latin-square design.

During infusions, the rats were gently restrained while the stylets were removed and 28-gauge bilateral injectors extending 5 or $2 \mathrm{~mm}$ beyond the length of the guide cannulae were inserted into the NAC or the dorsolateral striatum, respectively. A volume of $0.5 \mu \mathrm{l}$ was then infused bilaterally over 1 min into either the NAC or the dorsolateral striatum. The injector was left in place for $1 \mathrm{~min}$ to allow diffusion of the drug into the tissue surrounding the injector. The injector was then removed and the stylet replaced. After $5 \mathrm{~min}$, rats were tested in the 5CSRT.

Drugs were freshly prepared on each test day. SCH 23390, SKF 38393, quinpirole, and sulpiride were purchased from Research Biochemicals (Natick, MA) and dissolved in 0.9\% $\mathrm{NaCl}$. Sulpiride was dissolved in acidified $0.9 \% \mathrm{NaCl}$. The final $\mathrm{pH}$ was adjusted to approximately 7 using $0.1 \mathrm{M} \mathrm{NaOH}$.

\section{Experimental Design}

Experiment 1: performance on the 5CSRT following infusions of selective $D 1$ and D2 receptor agonists and antagonists into the NAC. Different groups of rats received infusions of vehicle and SCH $23390(1,10$, or $100 \mathrm{ng}$ per side; $n=11)$, of vehicle and SKF $38393(0.1,0.5$, or $1 \mu \mathrm{g}$ per side; $n=8)$, of vehicle and sulpiride $(0.1,1$, and $10 \mathrm{ng}$, per side; $n=9)$, or of vehicle and quinpirole $(0.1,0.5$, or $1 \mu \mathrm{g}$ per side; $n=8)$, according to a Latin square design. Rats were given standard sessions of the 5CSRT task with the same parameters as during training. Only rats with stable performance between 70 and $85 \%$ correct performance were employed to avoid ceiling effects (cf Granon et al, 2000). Doses of SCH 23390 and sulpiride were selected on the basis of pilot studies in order to avoid gross motor impairments in this strain of rats. The doses of quinpirole were chosen on the basis of previous experiments in our laboratory (Wolterink et al, 1993; Phillips et al, 1995). The partial D1 agonist SKF 38393 was chosen, rather than a full agonist as it was an aim to compare effects of intrastriatal infusions with previously published effects of SKF 38393 infused into the mPFC (Granon et al, 2000) on the same task. Furthermore, previous studies from our laboratory have already characterized the effects of NAC infusions of the same compound on other behavioral functions, which did not include gross motor effects (Wolterink et al, 1993; Phillips et al, 1995).

Experiment 2: performance on the 5CSRT following infusion of a high dose of the D1 receptor partial agonist SKF 38393 into the NAC. This experiment aimed to confirm that higher doses of the D1 receptor agonist in the NAC increases impulsive and perseverative responding as indicated by Experiment 1 . For this purpose, rats $(n=6)$ received infusions of vehicle and a high dose of SKF 38393 ( $5 \mu \mathrm{g}$ per side). Rats were subjected to standard sessions of the 5CSRT task with the same parameters as during training.

Experiment 3: effects of intra-NAC D1 receptor agonist SKF 38393 on 5CSRT performance with white noise distraction. In this experiment, the parameters of the task were modified to increase its attentional demands and to clarify further the nature of the improvement in accuracy observed after NAC infusion of the lowest dose of SKF $38393(0.1 \mu \mathrm{g} / \mathrm{side})$ in Experiment 1 . A burst of white noise $(0.5 \mathrm{~s}, 105 \mathrm{~dB})$ was presented $2.5,4.5$, or $5 \mathrm{~s}$ following the onset of the ITI. In the latter case, the white noise was presented simultaneously with the visual target stimulus. No noise was presented on $20 \%$ of the trials, these trials being randomly interspersed throughout the 30-min session. The introduction of distracting bursts of noise was intended to test whether intra-NAC infusions of SKF 38393 decreased rats' distractibility. The different trial types were presented in a randomized order and approximately 20 times each. This experiment was run as a betweensubjects design, so that each rat was exposed to the challenge for only one session. Sixteen rats were allocated to two groups matched for accuracy during training. One group received vehicle, the other group SKF $38393(0.1 \mu \mathrm{g}$ per side) before the session.

Experiment 4: neuroanatomical specificity of the effects $D 1$ or D2 receptor stimulation on 5CSRT performance effects of quinpirole and SKF 38393 in the dorsolateral striatum. To examine the neuroanatomical specificity of the effects observed in Experiments 1 and 2, one group of rats $(n=8)$ received infusions of vehicle $(0.9 \%$ saline $)$, SKF $38393(0.1 \mu \mathrm{g}$ per side), or quinpirole ( $1 \mu \mathrm{g}$ per side) into the dorsolateral striatum according to a Latin square design. Rats were subjected to standard sessions of the 5CSRT task with the same parameters as during training.

\section{Histology}

Following the completion of the experiments, subjects were anesthetized with a lethal dose of sodium pentobarbitone 
( $1.5 \mathrm{ml}$ sodium pentobarbitone Euthatal $(200 \mathrm{mg} / \mathrm{ml})$, Genus Express, UK) and perfused transcardially with $0.01 \mathrm{M}$ PBS followed by $4 \%$ paraformaldehyde. The brains were removed and postfixed in paraformaldehyde. Prior to being cut, the brains were transferred to $20 \%$ sucrose in $0.2 \mathrm{M}$ PBS and left overnight. Coronal sections were cut at $60 \mu \mathrm{m}$ on a freezing microtome and stained with Cresyl Violet. Cannulae locations were mapped onto standardized coronal sections of a rat brain stereotaxic atlas (Paxinos and Watson, 1998).

\section{Statistical Analysis}

The results are expressed as means $( \pm S E M)$ for each behavioral variable (ie percent correct, percent premature, percent perseverative responses, omissions, correct latency, magazine latency). Data were square-root, log, or arc-sine transformed prior to analysis to conform to the requirements for parametric statistics. In Experiments 1, 3, and 4, the data were subjected to ANOVA with dose as a withinsubjects factor. Effects revealed by the ANOVA were further analyzed by post hoc comparisons between means using Fisher's PLSD test. In Experiment 2, data were analyzed using ANOVA with dose as a between-subjects factor.

\section{RESULTS}

Experiment 1: Performance on the 5CSRT Following Infusions of Selective D1 and D2 Receptor Agonists and Antagonists into the NAC

Effects of the D1 receptor antagonist (SCH 23390) and agonist (SKF 38393). Intra-NAC infusion of SCH 23390 dose dependently affected the percentage of accurate responding $\left(\mathrm{F}_{(3,30)}=4.62 ; P<0.01\right)$, with the highest dose significantly decreasing accuracy as compared to the vehicle infusion (saline vs $100 \mathrm{ng} / \mathrm{side}, P<0.02$; all other doses $P>0.30$; Figure 1a). In parallel with this decrease in accurate responding, the highest dose of SCH 23390 also increased the number of omissions and the latency to make a correct response (omission: $\mathrm{F}_{(3,30)}=3.48 ; P<0.02$; saline vs $100 \mathrm{ng}, P<0.01$; correct latency: $\mathrm{F}_{(3,30)}=4.85$; $P<0.01 ; 100$ ng, $P<0.005$ ) (Figure 1a)). Neither impulsive $\left(\mathrm{F}_{(3,30)}=0.16 ; \quad P=0.91 ; \quad\right.$ Figure $\left.1 \mathrm{a}\right)$ nor perseverative $\left(\mathrm{F}_{(3,30)}=1.27 ; P=0.30\right.$; Figure $\left.1 \mathrm{~b}\right)$ responding nor any other performance measure $(\mathrm{F}<1.27, P>0.30)$ was affected by SCH 23390 .

Intra-NAC infusions of SKF 38393 improved accuracy at the lowest dose $\left(\mathrm{F}_{(3,21)}=3.61 ; P<0.04\right)$ (Figure $\left.1 \mathrm{~b}\right)$. Of the D1 receptor agonist, $0.1 \mu \mathrm{g} /$ side significantly increased the percentage of accurate responses as compared to vehicle infusions and as compared to the highest dose (saline vs

Figure I Effect of DI receptor blockade or stimulation in the nucleus accumbens on performance of the 5CSRT. (a) Choice accuracy, omissions, premature and perseverative responses, correct response latencies, and magazine latencies following bilateral intra-NAC infusions of saline or $\mathrm{SCH}$ 23390 (I, I0, or 100 ng/side). (b) Choice accuracy, omissions, premature and perseverative responses, correct response latencies, and magazine latencies following bilateral intra-NAC infusions of saline or SKF 38393 (0.1; 0.5 ; I $\mu \mathrm{g} / \mathrm{side})$. "Significant difference compared to saline $(P<0.05)$.
$0.1 \mu \mathrm{g}, P<0.03$; 0.1 vs $1 \mu \mathrm{g}, P<0.01$; saline vs $1 \mu \mathrm{g}, P=0.80$ ). The medium dose of $0.5 \mu \mathrm{g} / \mathrm{side}$ also tended to improve accuracy as compared to vehicle (saline $v s 0.5 \mu \mathrm{g}, P=0.07$ ).
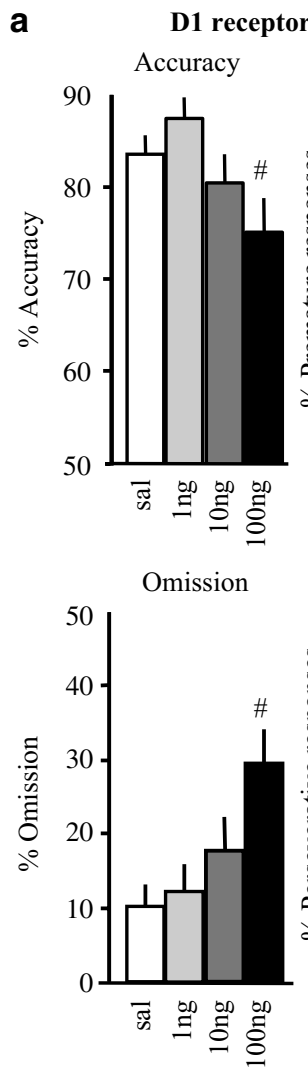

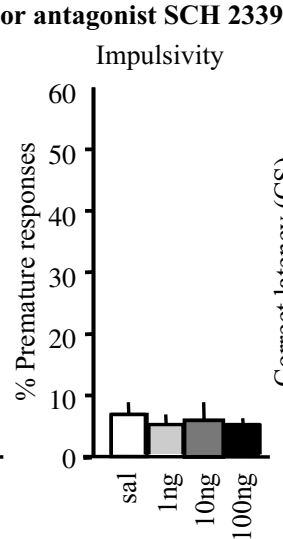

Perseveration

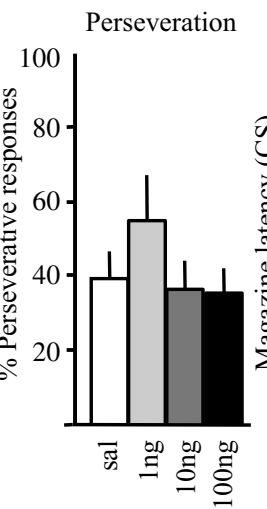

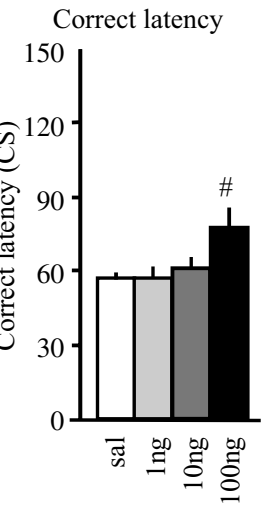

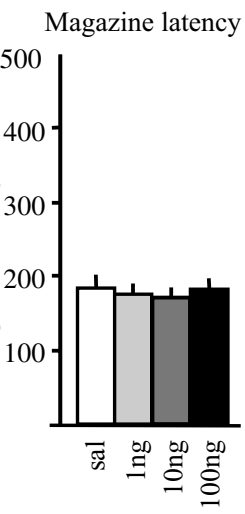

b D1 receptor agonist SKF 38393
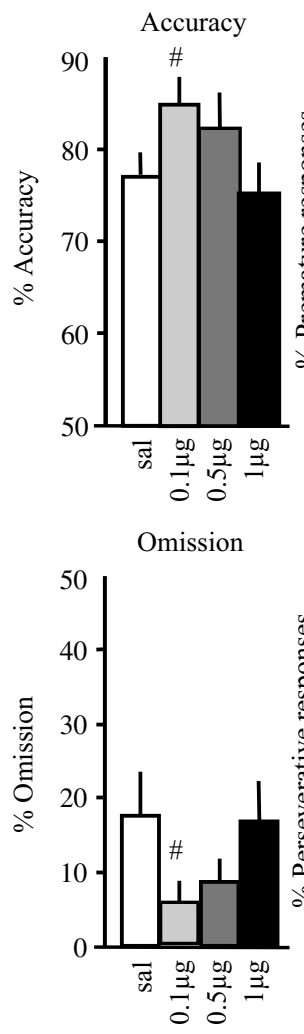
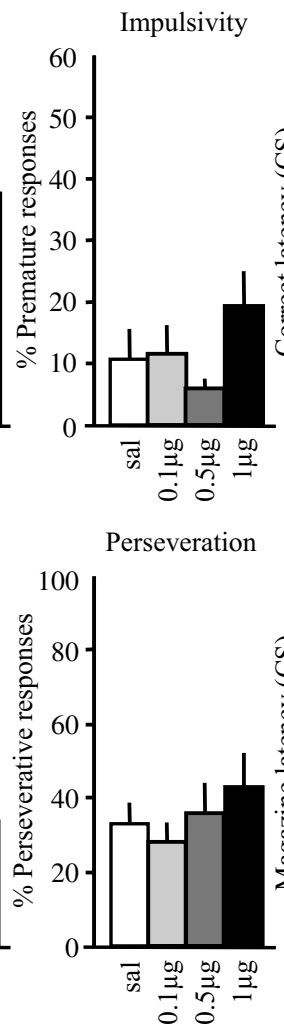

Correct latency

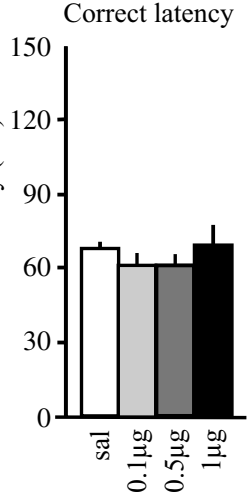

Magazine latency

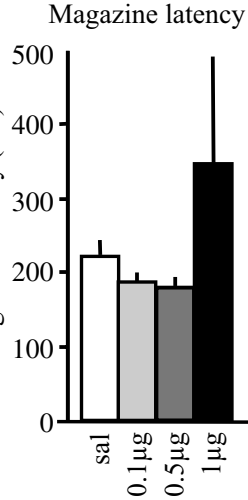


This increase in accuracy at the lowest dose of SKF 38393 was paralleled by a decrease in the percentage of omissions (Figure 1b) that approached statistical significance $\left(\mathrm{F}_{(3,21)}=2.90 ; P=0.06\right.$; saline vs $\left.0.1 \mu \mathrm{g}, P<0.02\right)$. There was a numerical increase in impulsive and perseverative responding at the highest dose $(1 \mu \mathrm{g} / \mathrm{side})$, which did not reach statistical significance (ANOVA for premature responding: $\mathrm{F}_{(3,21)}=1.74 ; P=0.19$; ANOVA for perseverative responding: $\mathrm{F}_{(3,21)}=2.83 ; P=0.07$; saline $v s 1 \mu \mathrm{g}$, $P=0.06$ ) (Figure $1 \mathrm{~b}$ ). None of the other measures of performance was affected (Figure 1b).

Effects of the D2 receptor antagonist (sulpiride) and agonist (quinpirole). Blockade of D2 receptors in the NAC by sulpiride reduced correct responses in a dose-dependent manner $\left(F_{(3,24)}=9.92 ; P<0.001\right.$; Figure $\left.2 \mathrm{a}\right)$, with only the highest dose yielding a significant effect (saline $v s 0.01 \mu \mathrm{g}$, $P<0.003$ ). In addition, the highest dose of sulpiride increased the percentage of omissions $\left(\mathrm{F}_{(3,24)}=21.36\right.$; $P<0.0001$; saline vs $10 \mathrm{ng}, P<0.0001)$, the latency to produce a correct response $\left(\mathrm{F}_{(3,24)}=11.88 ; P<0.0001\right.$; saline vs $10 \mathrm{ng}, P<0.0001)$, as well as the latency to collect the reward from the magazine $\left(\mathrm{F}_{(3,24)}=4.82 ; P<0.01\right.$; saline $v s 10 \mathrm{ng}, P<0.003$ ) (Figure 2a). None of the other measures of performance, such as impulsivity or perseverative responding, were significantly affected by sulpiride infusion $(\mathrm{F}<1.89 ; P>0.15$; Figure 2a).

In contrast to sulpiride, quinpirole infusions into the NAC had no effect on accuracy $\left(\mathrm{F}_{(3,21)}=0.07 ; P=0.97\right)$, premature responding $\left(\mathrm{F}_{(3,21)}=0.93 ; P=0.44\right)$, omissions $\left(\mathrm{F}_{(3,21)}=1.72 ; P=0.56\right)$, and latency to collect food from the magazine $\left(\mathrm{F}_{(3,21)}=0.69 ; P=0.44\right)$ (Figure $2 \mathrm{~b}$ ). However, perseverative responses were increased at all doses of the $\mathrm{D} 2$ receptor agonist $\left(\mathrm{F}_{(3,21)}=3.19 ; P<0.05\right.$; saline vs $0.1 \mu \mathrm{g}$, $P<0.001$; saline vs $0.5 \mu \mathrm{g}, P<0.04$; saline vs $1 \mu \mathrm{g}, P<0.04$ ) (Figure 2b). An increase in correct latency was observed to parallel this increase in perseverative responding $\left(\mathrm{F}_{(3,21)}=6.1 ; P=0.004\right.$; saline $v s 0.1 \mu \mathrm{g}, P=0.37$; saline $v s$ $0.5 \mu \mathrm{g}, P<0.05$; saline vs $1 \mu \mathrm{g}, P<0.01$ ) (Figure $2 \mathrm{~b}$ ).

\section{Experiment 2: Effects of a High Intra-NAC Dose of SKF 38393}

Infusing $5 \mu \mathrm{g} / \mathrm{side}$ of SKF 38393 into the NAC did not affect accuracy $\left(\mathrm{F}_{(1,10)}=0.53 ; P=0.49\right)$ but significantly increased premature responses $\left(\mathrm{F}_{(1,10)}=10.7 ; P<0.01\right)$ (Figure $3 \mathrm{a}$ ) with a strong trend towards increased perseverative responding $\left(\mathrm{F}_{(1,10)}=4.62, P=0.06\right)$. No other significant effects were observed for any other variable $(\mathrm{F}<4.89$; $P>0.08$; Figure 3a).

Figure 2 Effect of DA D2 receptor blockade or stimulation in the nucleus accumbens on performance of the 5CSRT. (a) Choice accuracy, omissions, premature and perseverative responses, correct response latencies, and magazine latencies following bilateral intra-NAC infusions of saline or sulpiride (0.1, I, or $10 \mathrm{ng} / \mathrm{side})$. (b) Choice accuracy, omissions, premature and perseverative responses, correct response latencies, and magazine latencies following bilateral intra-NAC infusions of saline or quinpirole $\left(0.1 ; 0.5 ; 1 \mu g /\right.$ side). ${ }^{\#}$ Significant difference compared to saline $(P<0.05)$.

\section{Experiment 3: Effects of Intra-NAC SKF 38393 on 5CSRT Performance with White Noise Distraction}

White noise presented at different times during the ITI did not significantly decrease percentage accuracy $\left(\mathrm{F}_{(1,7)}=3.11\right.$;

a D2 receptor antagonist sulpiride

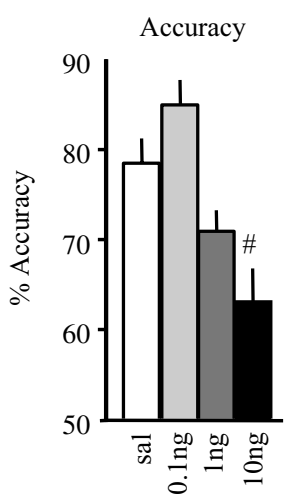

Impulsivity

Correct latency
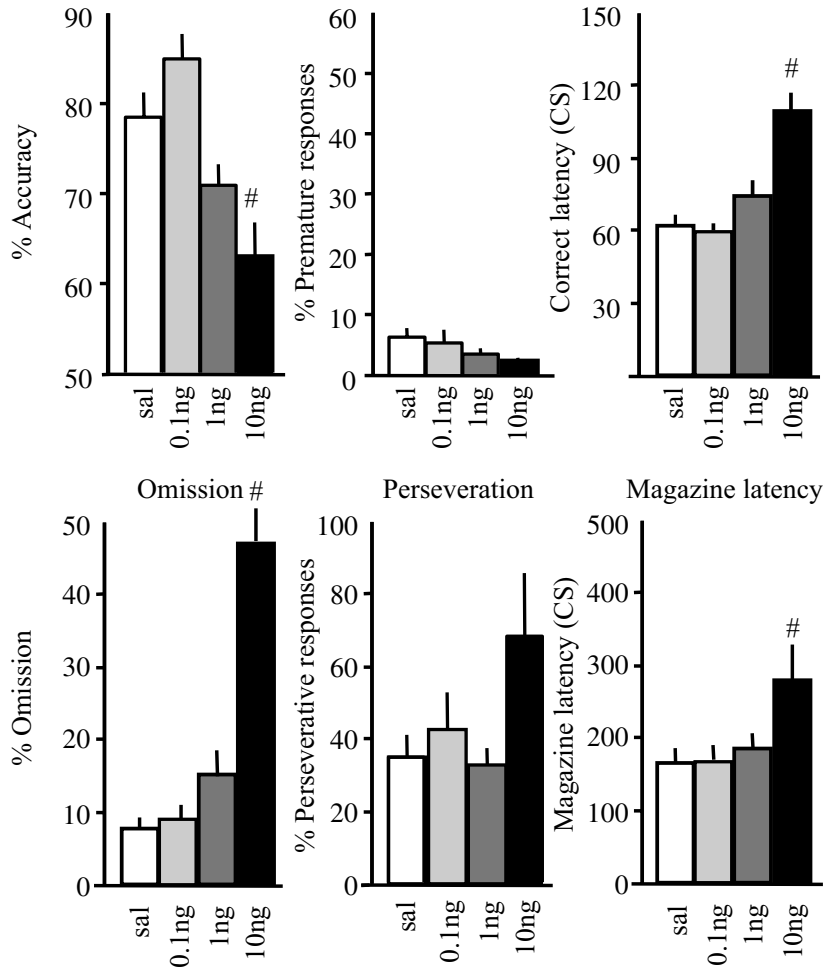

b

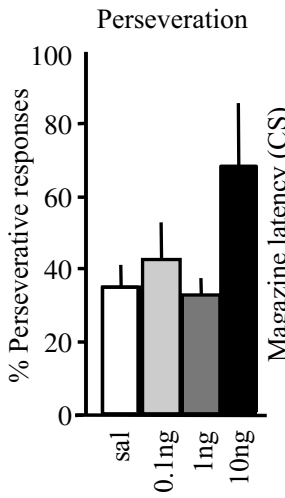

Magazine latency

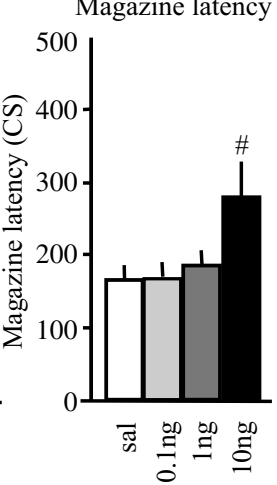

D2 receptor agonist quinpirole

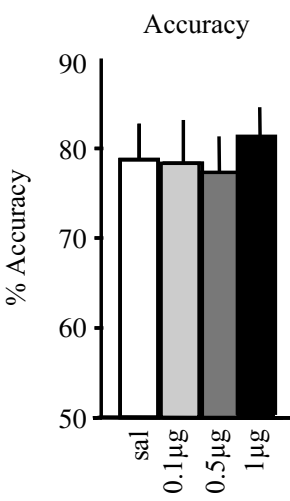

Impulsivity

Correct latency
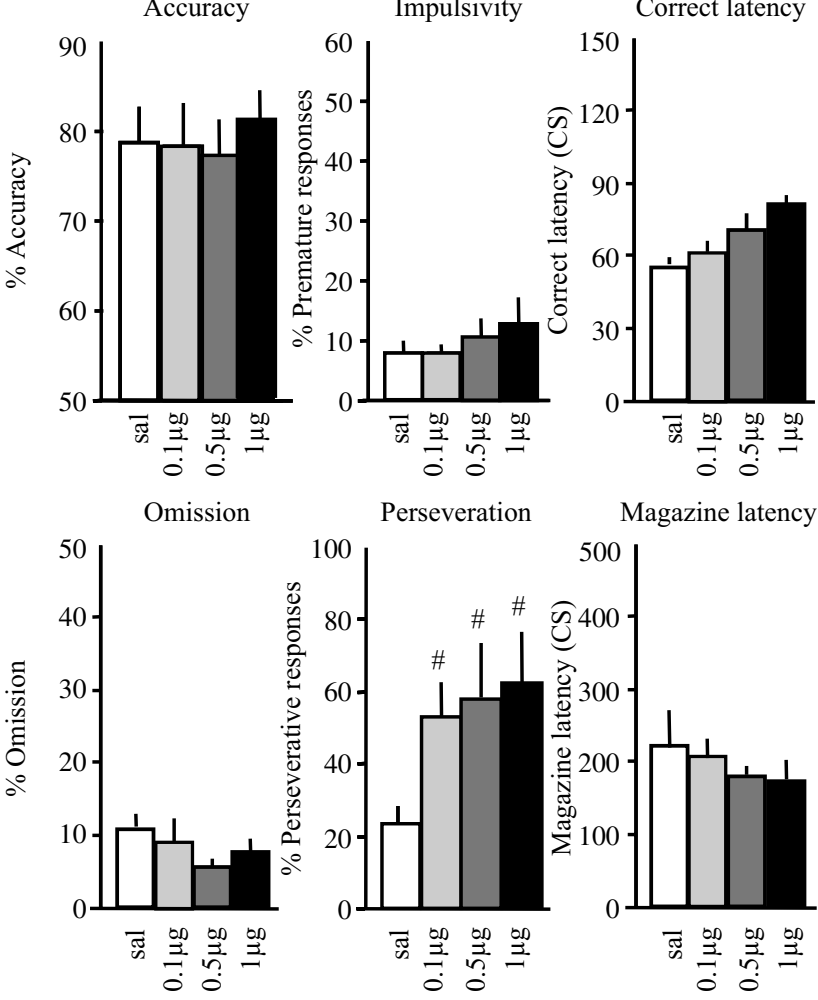

Perseveration

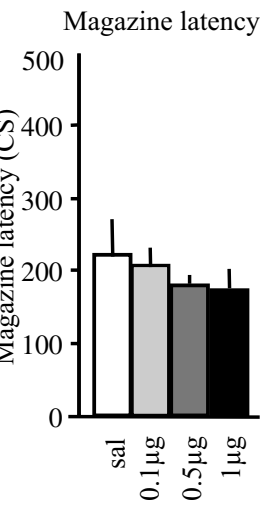


a

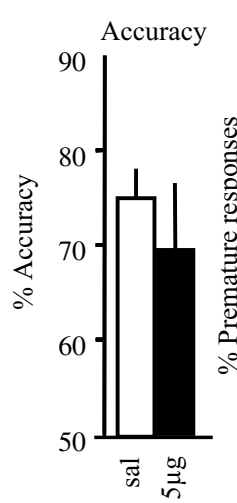

High dose of SKF 38393
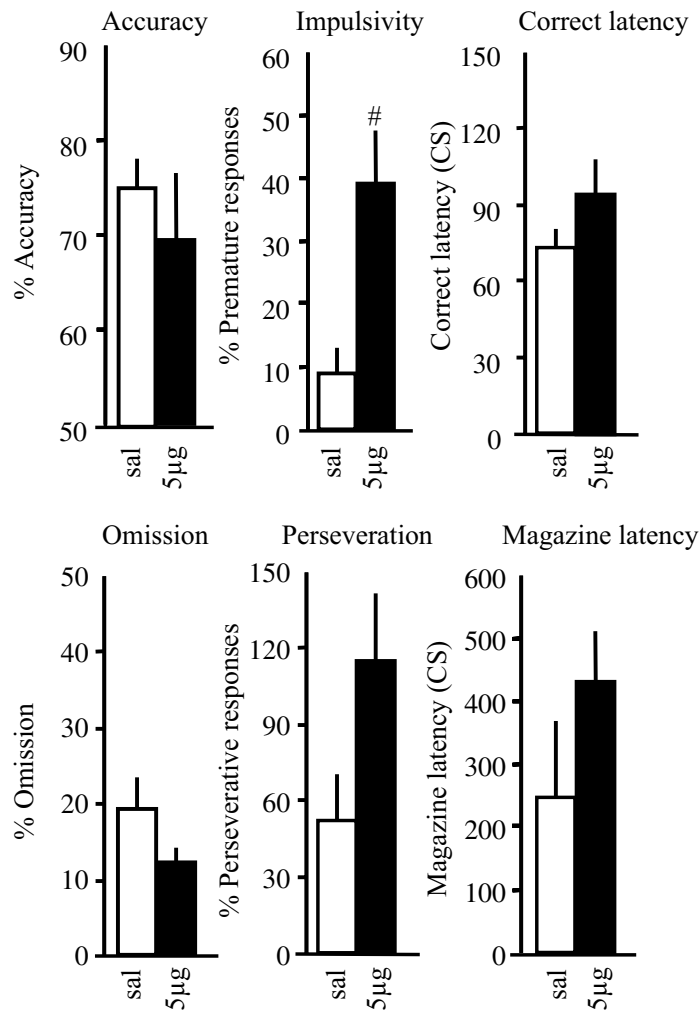

b

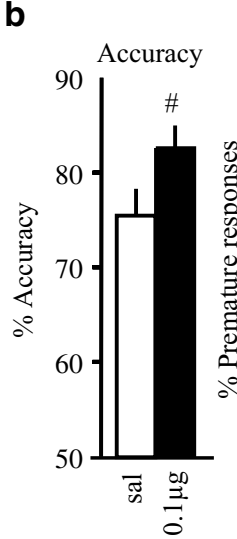

White noise distractor
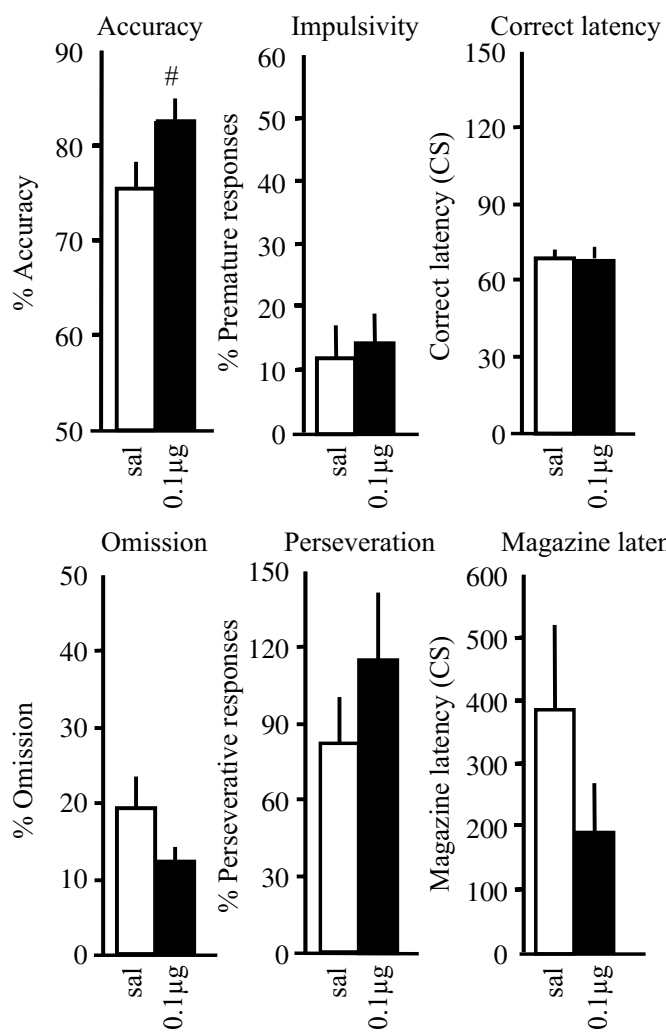

Figure 3 Effects of a higher intra-NAC dose of SKF 38393 ( $5 \mu \mathrm{g} / \mathrm{side})$ on 5CSRT performance. (a) Improved accuracy in the presence of a white noise distractor following intra-NAC infusions of SKF 38393 (0.1 $\mu \mathrm{g} / \mathrm{side}$ ). ${ }^{\#}$ Significant difference compared to saline $(P<0.05)$.
$P=0.12)$. There was, however, a large significant increase in the percentage of omissions $\left(\mathrm{F}_{(1,7)}=10.65 ; P<0.02\right)$ and in the latency to make correct responses $\left(\mathrm{F}_{(1,7)}=5.86\right.$; $P<0.05)$ as compared to the preceding baseline session. Similar to Experiment 1, infusions of SKF $38393(0.1 \mu \mathrm{g} /$ side) into the NAC produced a significant increase in the percentage of correct responses $\left(F_{(1,14)}=6.75 ; P<0.03\right)$ (Figure $3 \mathrm{~b}$ ) and a trend towards a decrease in omissions $\left(\mathrm{F}_{(1,14)}=3.63 ; \quad P=0.07\right)$. Neither impulsive responding $\left(\mathrm{F}_{(1,14)}=2.78 ; P=0.11\right.$; Figure $\left.3 \mathrm{~b}\right)$ nor any other variable were affected by SKF 38393 .

\section{Experiment 4: Neuroanatomical Specificity of the Effects Observed on 5CSRT Performance after D1 and D2 Receptor Stimulation: Effects of Intra-Dorso-Lateral Striatal SKF 38393 and Quinpirole}

No significant effect on percentage accuracy $\left(\mathrm{F}_{(2,12)}=1.23\right.$; $P=0.32)$, premature $\left(\mathrm{F}_{(2,12)}=0.63 ; P=0.54\right)$, and perseverative $\left(\mathrm{F}_{(2,12)}=2.76 ; P=0.10\right)$ responding was observed (Figure 4). While no significant effect on any other variable was observed, D2, but not D1, receptor stimulation in the dorsolateral striatum induced a significant increase in incorrect response latency $\left(\mathrm{F}_{(2,12)}=4.38 ; P<0.04\right.$; saline $v$ s $1 \mu$ g quinpirole, $P<0.03$ ).

\section{Effect of Intra-NAC Infusions of Saline on Basal Performance in the 5CSRT}

In Experiments 1, 2, and 4, none of the performance measures were significantly affected by vehicle infusions into the NAC as compared to the interinfusion baseline sessions (all $\mathrm{F}<0.9$ ).

\section{Histology}

Figure 5 shows reconstructed injector tip placements in the NAC or dorsolateral striatum, respectively, according to the atlas of Paxinos and Watson (1998). It can be seen that all injector tips were located throughout the NAC core region or the dorsolateral striatum between 1.2 and $2.2 \mathrm{~mm}$ anterior to bregma. No gross damage was seen after drug infusion, and the morphological structure of both the NAC and the dorsolateral striatum was preserved.

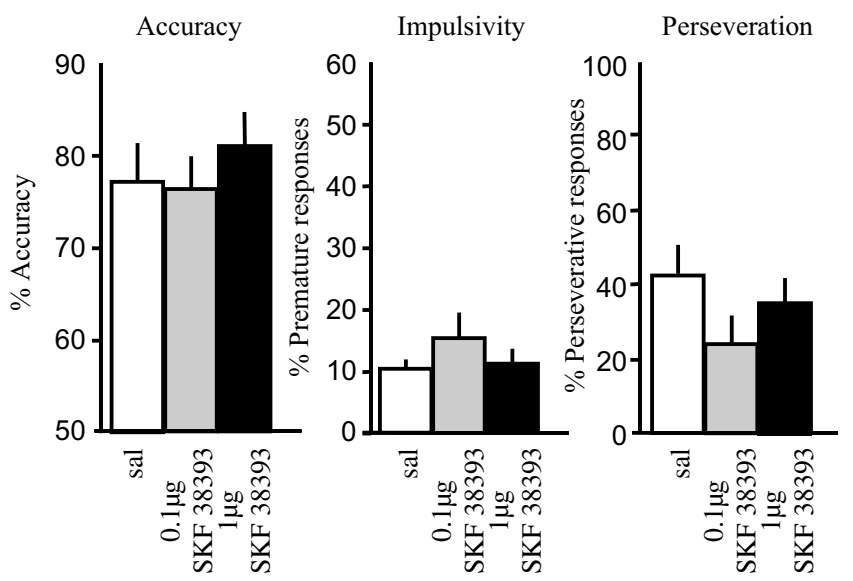

Figure 4 Lack of significant effects on 5CSRT performance of intradorsolateral striatal infusions of SKF 38393 (0.1 $\mu \mathrm{g} /$ side) and quinpirole ( $\mid \mu g /$ side). 


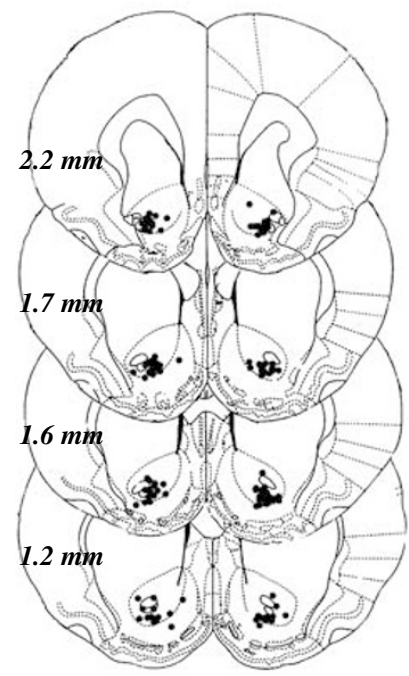

b

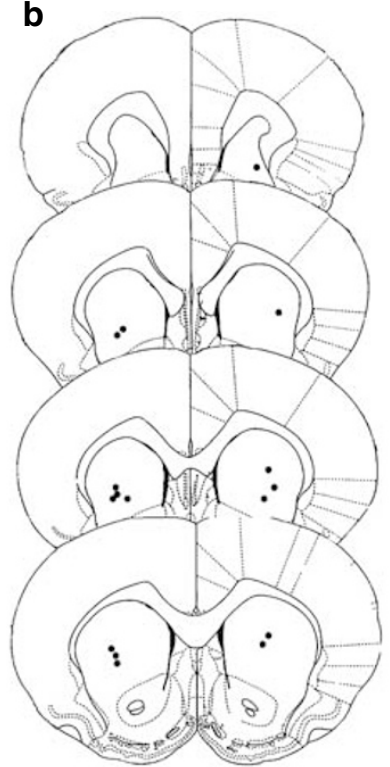

Figure 5 Bilateral placements of injector tips in the NAC (a) and dorsolateral striatum (b). Reconstructed from Paxinos and Watson (1998).

\section{DISCUSSION}

Infusions of DA receptor agents into the NAC had differential effects on 5CSRT performance according to DA receptor subtype. In two separate experiments, low doses of the D1 receptor partial agonist SKF 38393 improved the accuracy of visuo-spatial discrimination on the 5CRTT while also reducing errors of omission, whereas D1 receptor blockade (SCH 23390) (Experiment 1) decreased accuracy, increased omissions and slowed correct response latencies. The improved discrimination was not present at higher intra-NAC doses of the partial D1 receptor agonist which led instead to increases in premature, 'impulsive' responding. In contrast, D2 receptor stimulation in the NAC by quinpirole had no effect on discriminative accuracy, but increased perseverative responding and lengthened correct response latencies, while at the highest dose D2 receptor blockade (sulpiride) led to nonspecific impairments of overall performance, including a slowing of magazine latency. These findings indicate that D2 receptor stimulation in the NAC mostly modulates aspects of behavioral performance not primarily related to attention, including the control of perseverative responding. In contrast, attentional performance, as measured by discriminative accuracy in the 5CSRT, depends on an optimal level of D1 receptor stimulation in the NAC. These data are consistent with recent evidence of a similar distinction in the roles of these receptor subtypes in gating functions controlled by hippocampal or PFC inputs to the NAC (Goto and Grace, 2005).

\section{Neuroanatomical and Pharmacological Specificity}

Considering the small infusion volume and rate used in the present study $(0.5 \mu \mathrm{l}$ delivered over $1 \mathrm{~min})$, the estimated spread of the infused substances was about $1 \mathrm{~mm}$ (Myers, 1966; Myers et al, 1971; Routtenberg, 1972). Furthermore, to

demonstrate site specificity, we performed control experiments in which SKF 38393 and quinpirole were infused into the dorsolateral striatum at the doses that were most effective after NAC infusions. At these doses, neither SKF 38393 nor quinpirole produced the effects observed after NAC infusions. Thus, we conclude that the effects observed after our drug infusions into the NAC were mediated by actions on DA receptors within the NAC. The NAC contains two anatomically and functionally distinct subregions: a medioventral shell and a dorsolateral core (Zahm and Brog, 1992). The infusion sites were located in the NAC core, mainly ventral to the anterior commissure, and adjacent to the NAC shell subdivision. While we have shown that the observed effects did not result from a diffusion of our compounds into the dorsolateral striatum, a possible action of the drugs within the NAC shell cannot be excluded. It should be noted in particular that both quinpirole and sulpiride exhibit affinity for the D3 as well as the D2 receptor subtype of the D2 family (Levant, 1997), and D3 receptors are present in the NAC, especially the shell region (Schwartz et al, 2000). Hence, we cannot exclude the possibility of a D3 receptor involvement in the effects of sulpiride and quinpirole. SKF 38393 is also a partial agonist at the D1 receptor and so this possible action must be taken into account in explaining its effects. However, SCH 23390, a D1 receptor antagonist, had largely opposite effects on behavior in the 5CSRT, and so it seems reasonable to conclude that the effects of SKF 38393 were due to its agonist actions, as also concluded by Goto and Grace (2005).

\section{D1 Receptors in the NAC and 5CSRT Performance}

D1 receptor compounds yielded a pattern of effects on performance in the 5CSRT that differed in several aspects after D2 receptor manipulation. D1 receptor activation in the NAC by SKF 38393 enhanced attentional performance at lower doses, but increased premature responding at higher doses, while having no effect on perseverative responses. Intra-NAC infusion of the lowest dose of SKF $38393(0.1 \mu \mathrm{g} /$ side) decreased omissions in parallel with an increase in attentional selection, as reflected by increased accuracy. An improvement in accurate responding after intra-NAC infusion of SKF 38393 was also apparent when white noise distractors were presented during the ITI, although there was no evidence for a special action of SKF 38393 to protect performance from the effects of distraction. Overall, the ability of intra-NAC infusion of SKF 38393 to optimize performance could result from an improved ability to focus on task-relevant stimuli similarly to what has been shown for intra-PFC administration of SKF 38393 in poorly performing rats (Granon et al, 2000). It is of interest that the beneficial effect of D1 receptor stimulation is found at more than one node of the fronto-striatal network implicated in the control of 5CSRT performance, although we found no evidence for enhancement within the dorsolateral striatum (Experiment 4). This suggests that systemic treatment with appropriate D1 compounds may have therapeutic actions in a clinical context.

Much evidence shows that similar improvements in 5CSRT performance can be achieved via modulation of cholinergic projections to the PFC from the basal forebrain 
(Muir et al, 1994; McGaughy et al, 2002; Lehmann et al, 2003; Dalley et al, 2004). Indeed, it has recently been demonstrated that NMDA and DA interactions in the NAC modulate cortical acetylcholine (Zmarowski et al, 2005). It is thus possible that the present enhancement of 5CSRT performance via NAC D1 receptor stimulation is mediated in part via serial interactions of NAC outflow with this basal forebrain cholinergic system (Ingham et al, 1988; Moore et al, 1999; Sarter et al, 1999).

The beneficial effects of the partial D1 receptor agonist were different from those of intra-NAC D-amphetamine, which mainly increased premature responding (Cole and Robbins, 1987), like the higher dose of SKF 38393. The lack of effect on discrimination may reflect additional actions of intra-NAC D-amphetamine on other receptors, such as D2. For example, D1 and D2 receptor effects may partly oppose each other due to their hyperpolarizing and depolarizing actions, respectively, on the membrane potential of NAC medium spiny neurons (Uchimura et al, 1986; Pennartz et al, 1994; Nicola et al, 2000). The effects of SKF 38393 on performance of the 5CSRT also contrasted with those of the D1 antagonist, consistent with the mediation of the effects of both drugs by opposite actions at D1 receptors. Finally, in contrast to D2 receptor blockade, D1 receptor blockade did not impair primary motivation, as there were no significant effects on other relevant variables, such as magazine latency to collect a food pellet.

In a previous study, 6-hydroxydopamine (6-OHDA)induced depletion (by 87\%) of DA in the NAC attenuated both the speed and impulsivity of responding in the baseline condition of the 5CSRT (Cole and Robbins, 1989). Consistent with these observations, we showed here that SCH 23390 and SKF 38393 infused into the NAC increased and decreased the latency to produce correct responses, respectively. Furthermore, while intra-NAC infusions of SCH 23390 did not significantly alter the number of premature responses, SKF 38393 increased impulsivity, at the highest dose (resembling the effects of intra-NAC D-amphetamine, Cole and Robbins, 1987). There was only a nonsignificant tendency for accuracy to be decreased by NAC 6-OHDA (Cole and Robbins, 1989) but the acute dopaminergic manipulations in the present study produced significant deficits. The most likely explanations for reconciling these results are (i) the accuracy measures are less sensitive indicators of deficits than those affecting speed and impulsivity following manipulations of DA-ergic activity in the NAC or (ii) in terms of differences between permanent and acute manipulations. Thus, long-lasting DA depletion within the NAC, in contrast to temporary DA receptor blockade, may have failed to affect discriminative accuracy because of compensatory processes triggered by such permanent lesions (see also Schoenfeld and Hamilton, 1977; Lomber, 1999; Bast and Feldon, 2003). Finally, (iii) the present study tested the behavioral effects of selective dopamine receptor agents. A previous study (Cole and Robbins, 1987) showed no significant effect of the mixed DA receptor antagonist alpha-flupenthixol administered systemically on the accuracy of 5CSRT performance. However, targeting specific DA receptor subtypes within the NAC, as in this study, may account for the lack of effects on accuracy found previously after overall DA receptor blockade, or NAC DA depletion.
In previous studies it has been demonstrated that DA release in the NAC promotes appropriate behavioral responses to reward-predictive cues (Yun et al, 2004; Nicola et al, 2005). In line with a dissociation of D1 vs D2 receptor functions, Floresco and Phillips (1999) have shown that D1 but not D2 receptor blockade in the NAC impaired accurate responding during foraging behavior. Our study demonstrates that the selection of appropriate behavioral responses is supported by D1 receptor stimulation in the NAC. The effect of the D1 receptor agonist to increase premature responding and reduce the number of omissions, is consistent with a general response-rate invigorating effect of enhanced mesolimbic DA activity, which also occurs, for example, following intra-NAC D-amphetamine (Taylor and Robbins, 1986; Parkinson et al, 1999). The parallel increase in accurate responding implies a greater selectivity in responding to the appropriate rewarded cue and thus, may reflect a separate effect to heighten 'incentive salience' (Wyvell and Berridge, 2000, 2001). The finding in Experiment 3 that D1 receptor stimulation in the NAC continues to enhance attentional performance even after the introduction of a noise distractor strengthens this interpretation. The role of NAC D1 receptors on response selection is evidently not restricted to attentional performance, as recent data have shown that D1 receptors are implicated in the consolidation of discriminative approach behavior (Dalley et al, 2005).

Cognitive functions, such as working memory and attention, are related to an optimal level of D1 receptor stimulation in the mPFC (Sawaguchi and Goldman-Rakic, 1991; Zahrt et al, 1997; Arnsten, 1997; Granon et al, 2000; Chudasama and Robbins, 2004b; Pezze et al, 2003). Similarly to these studies with intra-PFC manipulations, we have shown here for the NAC that the greatest enhancement in accuracy was observed at the lowest dose of SKF 38393, while infusions of higher doses had little effect on accuracy. These data indicate that an inverted U-shaped function relates D1 receptor stimulation in the NAC to the efficiency of attentional performance (Zahrt et al, 1997; Arnsten, 1997). In both cases the beneficial effects of D1 receptor stimulation may be limited by the emergence of additional effects on behavior at higher doses: for example, increased perseveration in delayed alternation tasks (Zahrt et al, 1997) and increased 'impulsive' responding in this study.

Recently, it has been shown that both behavioral responses to cue-mediated reward and the specific NAC neuronal firing promoting such responses, depends on DA release within the NAC (Nicola et al, 2004; Yun et al, 2004). In addition, it has been observed that in NAC medium spiny neurons, D1 receptor stimulation has the dual effect of depolarizing the cell to the firing threshold and of reducing the efficacy of the weakest synaptic inputs (Nicola et al, 2000; O'Donnell, 2003). The attentional effects of NAC D1 receptor stimulation in the 5CSRT observed in the present study may thus be one behavioral correlate of this physiological mechanism.

\section{Role of NAC D2 Receptors in Attentional Performance}

D2 receptor blockade in the NAC resembled many of the effects of intra-NAC D1 receptor blockade, including 
reduced accuracy, increased omissions and slower response latencies. Thus, a role for NAC D2 receptors in attentional discrimination cannot be entirely ruled out. However, in addition to these effects, sulpiride also increased magazine latencies, a measure most obviously reflecting primary motivation (Robbins, 2002). Therefore, although SCH 23390 and sulpiride produced somewhat similar deficits in attentional performance these may be mediated by different underlying processes.

Intra-NAC infusions of the D2 receptor agonist quinpirole increased perseverative responding, even at the lowest dose, and also affected the latency to make a correct response at higher doses. Considered together, these impairments possibly reflect an inability to coordinate behavioral sequencing in order to optimize performance (Chudasama et al, 2003). In this respect, the increased latency to respond correctly and the increase in perseveration suggest that the rats are unable to shift from the initiation of the trial to the response, and from a previous response to the initiation of a new trial, respectively. Excitotoxic lesions of the NAC as well as the Cg3 region of the $\mathrm{mPFC}$ and the orbitofrontal cortex induced similar deficits in perseverative responding as measured by the 5CSRT (Christakou et al, 2004; Chudasama et al, 2003; Chudasama and Muir, 2001). NAC DA has been proposed to be a key element in guiding the switch between sequential steps of a complex behavior (Redgrave et al, 1999; Pezze and Feldon, 2004). Our results are in agreement with this notion and go further in demonstrating that such response selection mechanisms are impaired if D2 receptor stimulation within the NAC is excessive.

The findings are also consistent with the effects of disconnection lesions of the MPFC and the core subregion of the NAC which have been shown to affect similar aspects of response control (Christakou et al, 2004). Unilateral lesions of the mPFC and of the NAC in the opposite hemisphere induced increases in perseverative responses in the 5CSRT. Thus, such cortico-striatal projections may influence the switch of a response to the next step of a behavioral sequence. It has been shown in vitro that NAC excitatory responses induced by $\mathrm{PFC}$ stimulation are decreased by the administration of the DA D2 receptor agonist quinpirole (O'Donnell and Grace, 1994). Brady and O'Donnell (2004) have shown that the ability of DA inputs to decrease prefrontal stimulation of the NAC neurons is blocked by the D2 antagonist eticlopride, suggesting the necessity of D2 receptors in mediating this response. Moreover, Goto and Grace (2005) have recently argued that PFC-mediated set shifting behavior is impaired by D2 receptor stimulation within the NAC. Thus, an overstimulation of D2 receptors in the NAC may shut down mPFC inputs to the NAC, and this effect may underlie the perseverative responding observed in the present study after intra-NAC quinpirole, which may provide a model of 'compulsive' behavior (see Chudasama et al, 2003).

\section{Conclusion}

The present study demonstrated that optimal performance on the 5CSRT depends on both D1 and D2 receptors in the NAC, but they modulate different aspects of performance. D2 receptor transmission within the NAC was more involved in those aspects of response control that enable switching between elements in a behavioral sequence, and in the primary motivation necessary for task performance. In contrast, D1 receptor transmission in the NAC modulated the accuracy of discrimination in a continuous test of attentional performance.

\section{ACKNOWLEDGEMENTS}

The research was supported by a Programme grant from the Wellcome Trust to TWR, BJ Everitt, AC Roberts, and BJ Sahakian, and completed within the University of Cambridge Behavioural and Clinical Neuroscience Institute, supported by a joint award from the Medical Research Council and the Wellcome Trust. MP was supported by a Marie-Curie Programme Personal Fellowship. We thank David Theobald for technical assistance, Rudolf Cardinal for software programming, Olivia Lehmann for sharing her knowledge of the 5CSRT, and Tobias Bast for discussion and reading of the manuscript.

\section{REFERENCES}

Arnsten AFT (1997). Cathecholamine regulation of the prefrontal cortex. J Psychopharmacolol 11: 151-162.

Bast T, Feldon J (2003). Hippocampal modulation of sensorimotor processes. Prog Neurobiol 70: 319-345.

Baunez C, Robbins TW (1999). Effects of dopamine depletion of the dorsal striatum and further interaction with the subthalamic nucleus lesions in an attentional task in the rat. Neuroscience 92: 1343-1356.

Berridge KC, Robinson TE (1998). What is the role of dopamine in reward: hedonic impact, reward learning, or incentive salience? Brain Res Brain Res Rev 28: 309-369.

Brady AM, O'Donnell P (2004). Dopaminergic modulation of prefrontal cortical input to nucleus accumbens neurons in vivo. J Neurosc 24: 1040-1049.

Carli M, Robbins TW, Evenden JL, Everitt BJ (1983). Effects of lesions to ascending noradrenergic neurones on performance of a 5-choice serial reaction task in rats; implications for theories of dorsal noradrenergic bundle function based on selective attention and arousal. Behav Brain Res 9: 361-380.

Christakou A, Robbins TW, Everitt BJ (2004). Prefrontal corticalventral striatal interactions involved in affective modulation of attentional performance: implication for corticostriatal circuit function. J Neurosci 24: 773-780.

Chudasama Y, Muir JL (2001). Visual attention in the rat: a role for the prelimbic cortex and thalamic nuclei. Behav Neurosci 115: 417-428.

Chudasama Y, Passetti F, Rhodes SE, Lopian D, Desai A, Robbins TW (2003). Dissociable aspects of performance on the 5-choice serial reaction time task following lesions of the dorsal anterior cingulate, infralimbic and orbitofrontal cortex in the rat: differential effects on selectivity, impulsivity and compulsivity. Behav Brain Res 146: 105-119.

Chudasama Y, Robbins TW (2004a). Psychopharmacological approaches to modulating attention in the five-choice serial reaction time task: implications for schizophrenia. Psychopharmacology (Berlin) 174: 86-98.

Chudasama Y, Robbins TW (2004b). Dopaminergic modulation of visual attention and working memory in the rodent prefrontal cortex. Neuropsychopharmacology 29: 1628-1636.

Cole BJ, Robbins TW (1987). Amphetamine impairs the discrimination performance of rats with dorsal bundle lesions on a 5-choice serial reaction time task: new evidence for central 
dopaminergic-noradrenergic interactions. Psychopharmacology 91: 458-466.

Cole BJ, Robbins TW (1989). Effects of 6-hydroxydopamine lesions of the nucleus accumbens septi on performance of a 5-choice serial reaction time task in rats: implications for theories of selective attention and arousal. Behav Brain Res 33: 165-179.

Dalley JW, Laane K, Theobald DHC, Armstrong HC, Corlett PR, Chudasama $\mathrm{Y}$ et al (2005). Time-limited modulation of appetitive learning by D1 and NMDA receptors in the nucleus accumbens. Proc Natl Acad Sci 17: 6189-6194.

Dalley JW, Theobald DE, Bouger P, Chudasama Y, Cardinal RN, Robbins TW (2004). Cortical cholinergic function and deficits in visual attentional performance in rats following 192 IgGsaporin-induced lesions of the medial prefrontal cortex. Cereb Cortex 14: 922-932.

Floresco SB, Phillips AG (1999). Dopamine and hippocampal input to the nucleus accumbens plays an essential role in search for food in an unpredictable environment. Psychobiology 27: 277-286.

Floresco SB, Seamans JK, Phillips AG (1996). A selective role for dopamine in the nucleus accumbens of the rat in random foraging but not delayed spatial win-shift-based foraging. Behav Brain Res 80: 161-168.

Goldman-Rakic PS, Funahashi S, Bruce CJ (1990). Neocortical memory circuits. Cold Spring Harbor Symp Quant Biol 55: $1025-1472$.

Goto Y, Grace A (2005). Dopamine modulation of limbic and cortical drive of nucleus accumbens in goal-directed behavior. Nat Neurosc 8: 805-812.

Granon S, Passetti F, Thomas KL, Dalley JW, Everitt BJ, Robbins TW (2000). Enhanced and impaired attentional performance after infusion of D1 dopaminergic receptor agents into rats prefrontal cortex. J Neurosci 20: 1208-1215.

Groenewegen HJ, Wright CI, Beijer AVJ, Voorn P (1999). Convergence and segregation of ventral striatal inputs and outputs. Ann NY Acad Sci 877: 49-63.

Himmelheber AM, Bruno JP, Starter M (2000). Effects of intraaccumbens infusions of amphetamine or cis-flupenthixol on sustained attention performance in rats. Behav Brain Res 116: 123-133.

Ingham CA, Bolam JP, Smith AD (1988). GABA-immunoreactive synaptic boutons in the rat basal forebrain: comparison of neurons that project to the neocortex with pallidosubthalamic neurons. J Comp Neurol 273: 263-282.

Lehmann O, Grottick AJ, Cassel JC, Higgins GA (2003). A double dissociation between serial reaction time and radial maze performance in rats subjected to 192 IgG-saporin lesions of the nucleus basalis and/or the septal region. Eur J Neurosci 18: 651-666.

Levant B (1997). The D3 dopamine receptor: neurobiology and potential clinical relevance. Pharmacol Rev 49: 231-252.

Lomber SG (1999). The advantages and limitations of permanent or reversible deactivation techniques in the assessment of neural function. J Neurosci Meth 86: 109-117.

McGaughy J, Dalley JW, Morrison CH, Everitt BJ, Robbins TW (2002). Selective behavioral and neurochemical effects of cholinergic lesions produced by intrabasalis infusions of 192 IgG-saporin on attentional performance in a five choice serial reaction time task. J Neurosci 22: 1905-1913.

Miner LA, Sarter M (1999). Intra-accumbens infusions of antisense oligonucleotides to one isoform of glutamatergic acid decarboxylase mRNA, GAD65 but not to GAD67, impaired substained attention in the rat. Cog Brain Res 7: 269-283.

Moore H, Fadel J, Sarter M, Bruno JP (1999). Role of accumbens and cortical dopamine receptors in the regulation of cortical acetylcholine release. Neuroscience 88: 811-822.

Muir JL, Everitt BJ, Robbins TW (1994). AMPA-induced excitotoxic lesions of the basal forebrain: a significant role for the cortical cholinergic system in attentional function. J Neurosci 14: 2313-2326.

Muir JL, Everitt BJ, Robbins TW (1996). The cerebral cortex of the rat and visual attentional functions: dissociable effects of the mediofrontal, cingulate, anterior dorsolateral, and parietal cortex lesion on a five-choice serial reaction time task. Cereb Cortex 6: 470-481.

Myers RD (1966). Injection of solutions into cerebral tissue: relation between volume and diffusion. Physiol Behav 1: 171-174.

Myers RD, Tytell M, Kawa A, Rudy T (1971). Micro-injection of $3 \mathrm{H}$-acetylcholine, $14 \mathrm{C}$-serotonin and $3 \mathrm{H}$-norepinephrine into the hypothalamus of the rat: diffusions into tissue and ventricles. Physiol Behav 7: 743-751.

Nicola SM, Surmeier J, Malenka RC (2000). Dopamine modulation of neuronal excitability in the striatum and nucleus accumbens. Annu Rev Neurosci 23: 185-215.

Nicola SM, Taha SA, Kim SW, Fields HL (2005). Nucleus accumbens dopamine release is necessary and sufficient to promote the behavioral response to reward-predictive cues. Neuroscience 135: 1025-1033.

Nicola SM, Yun IA, Wakabayashi KT, Fields HL (2004). Cueevoked firing of nucleus accumbens neurons encodes motivational significance during a discriminative stimulus task. $J$ Neurophysiol 91: 1840-1865.

O'Donnell P (2003). Dopamine gating of forebrain neuronal ensembles. Eur J Neurosci 17: 429-435.

O'Donnell P, Grace AA (1994). Tonic D2-mediated attenuation of cortical excitation in nucleus accumbens neurons recorded in vitro. Brain Res 634: 105-112.

Parkinson JA, Olmstead MC, Burns LH, Robbins TW, Everitt BJ (1999). Dissociation in effects of lesions of the nucleus accumbens core and shell on appetitive pavlovian approach behavior and the potentiation of conditioned reinforcement and locomotor activity by D-amphetamine. J Neurosci 19: 2401-2411.

Passetti F, Chudasama Y, Robbins TW (2002). The frontal cortex of the rat and visual attention performance: dissociable functions of distinct medial prefrontal subregions. Cereb Cortex 12: $1254-1268$.

Paxinos G, Watson C (1998). The Rat Brain in Stereotaxic Coordinates, 4th edn. Academic Press: New York.

Pennartz CM, Groenewegen HJ, Lopes da Silva FH (1994). The nucleus accumbens as a complex of functionally distinct neuronal ensembles: an integration of behavioural, electrophysiological and anatomical data. Prog Neurobiol 42: 719-761.

Pezze MA, Bast T, Feldon J (2003). Significance of dopamine transmission in the medial prefrontal cortex for conditioned fear. Cereb Cortex 13: 371-380.

Pezze MA, Feldon J (2004). Mesolimbic dopaminergic pathways in fear conditioning. Prog Neurobiol 74: 301-320.

Phillips GD, Howes SR, Whitelaw RB, Robbins TW, Everitt BJ (1995). Analysis of the effects of intra-accumbens SKF-38393 and LY-171555 upon the behavioural satiety sequence. Psychopharmacology 117: 82-90.

Redgrave P, Prescott TJ, Gurney K (1999). Is the short-latency dopamine response too short to signal reward error? Trends Neurosci 22: 146-151.

Robbins TW (2000). From arousal to cognition: the integrative position of the prefrontal cortex. Prog Brain Res 126: 469-483.

Robbins TW (2002). The 5-choice serial reaction time task: behavioural pharmacology and functional neurochemistry. Psychopharmacology 163: 362-380.

Robbins TW, Muir JL, Killcross AS, Pretsell D (1993). Methods for assessing attention and stimulus control in the rat. In: Sahgal A (ed). Behavioral Neuroscience: A Practical Approach. Oxford University Press: Oxford. pp 13-48.

Rogers R, Baunez C, Everitt BJ, Robbins TW (2001). Lesions of the medial and lateral striatum in the rat produce dissociable 
deficits in attentional performance. Behav Neurosci 115: 799-811.

Routtenberg A (1972). Intracranial chemical injection and behavior: a critical review. Behav Biol 7: 601-641.

Sarter M, Bruno JP, Turchi J (1999). Basal forebrain afferent projections modulating cortical acetylcholine, attention, and implications for neuropsychiatric disorders. Ann NY Acad Sci 877: 368-382.

Sawaguchi T, Goldman-Rakic PS (1991). D1 dopamine receptors in prefrontal cortex: involvement in working memory. Science 251: 947-950.

Schoenfeld TA, Hamilton LW (1977). Secondary brain changes following lesions: a new paradigm for lesion experimentation. Physiol Behav 18: 951-967.

Schwartz J-C, Diaz J, Pilon C, Sokoloff P (2000). Possible implications of the dopamine D3 receptor in schizophrenia and in anti-psychotic drug actions. Brain Res Rev 31: 277-287.

Taylor JR, Robbins TW (1986). 6-Hydroxydopamine lesions of the nucleus accumbens, but not of the caudate nucleus, attenuate enhanced responding with reward-related stimuli produced by intra-accumbens D-amphetamine. Psychopharmacology 90: 390-397.

Uchimura N, Higashi H, Nishi S (1986). Hyperpolarizing and depolarizing actions of dopamine via D-1 and D-2 receptors on nucleus accumbens neurons. Brain Res 375: 368-372.
Wolterink G, Phillips G, Cador M, Donselaar-Wolterink I, Robbins TW, Everitt BJ (1993). Relative roles of ventral striatal D1 and D2 dopamine receptors in responding with conditioned reinforcement. Psychopharmacology 110: 355-364.

Wyvell CL, Berridge KC (2000). Intra-accumbens amphetamine increases the conditioned incentive salience of sucrose reward: enhancement of reward 'wanting' without enhanced 'liking' or response reinforcement. J Neurosci 20: 8122-8130.

Wyvell CL, Berridge KC (2001). Incentive sensitization by previous amphetamine exposure: Increase cue-triggered 'wanting' for sucrose reward. J Neurosci 20: 8122-8130.

Yun IA, Wakabayashi KT, Fields HL, Nicola SM (2004). The ventral tegmental area is required for the behavioral and nucleus accumbens neuronal firing responses to incentive cues. $J$ Neurosci 24: 2923-2933.

Zahm DS, Brog JS (1992). On the significance of subterritories in the 'accumbens' part of the rat ventral striatum. Neuroscience 50: 751-767.

Zahrt J, Taylor JR, Mathew RG, Arnsten AFT (1997). Supranormal stimulation of D1 dopamine receptors in the rodent prefrontal cortex impairs working memory performance. J Neurosci 17: 8528-8535.

Zmarowski A, Sarter M, Bruno JP (2005). NMDA and dopamine interactions in the nucleus accumbens modulate cortical acethylcholine release. Eur J Neurosci 17: 1731-1740. 